\title{
MANEUVERING TARGET ALTITUDE TRACKING IN OVER-THE-HORIZON RADARS EXPLOITING MULTIPATH DOPPLER SIGNATURES
}

\author{
Yimin D. Zhang ${ }^{\dagger}$, Jun Jason Zhang ${ }^{\ddagger}$, Moeness G. Amin ${ }^{\dagger}$, and Braham Himed ${ }^{\S}$ \\ $\dagger$ Center for Advanced Communications, Villanova University, Villanova, PA 19085, USA \\ ¥ Electrical and Computer Engineering Department, University of Denver, Denver, CO 80210, USA \\ $\S$ RF Technology Branch, Air Force Research Lab (AFRL/RYMD), WPAFB, OH 45433, USA
}

\begin{abstract}
Over-the-horizon radar (OTHR) systems provide wide-area surveillance capabilities to detect and track targets far beyond the range of conventional line-of-sight radars. Because of the narrowband waveforms, OTHR systems do not achieve reliable altitude estimation. In this paper, we develop a new technique to track the instantaneous altitude of maneuvering targets by exploiting the estimated multicomponent Doppler signatures. The main contribution of this paper is to apply effective non-stationary signal analysis for estimating the time-varying Doppler signature of each individual multipath, which is then applied to an extended Kalman filter to reliably track the instantaneous target altitude.
\end{abstract}

Index Terms - Over-the-horizon radar, target tracking, Kalman filter, time-frequency analysis, multipath exploitation

\section{INTRODUCTION}

Skywave over-the-horizon radar (OTHR) systems exploit highfrequency (HF) waveforms to propagate through reflection and refraction from the ionosphere and, as such, provide wide-area surveillance capabilities to detect and track targets far beyond the range of conventional line-of-sight radars [1, 2]. OTHR systems use narrowband signals due to the constraints of the ionospheric conditions and the range extents. As such, the range resolution of an OTHR system is typically measured in tens of kilometers [2]. Such range resolution is very useful in practice considering the long range of the targets, but it is not the case for the target altitude. For the target altitude estimate to be informative for the determination of the type and flying course of a target, an accuracy of a few kilometers or less is required. Toward this end, micro-multipath model, which exploits multipath returns due to earth reflections that are local to the target, can be devised to provide useful altitude information [3].

The micro-multipath propagation is illustrated in Fig. 1 using a simplified flat-earth propagation model [4]. We focuses on the relatively stable E-layer. Assume that, in addition to the target motion in the range direction, which generates a nominal Doppler signature, the target ascends in altitude. In this case, changes in the target altitude will alter the distance of both paths, generating Doppler variations with opposite signs around the nominal Doppler signature

The work of Y. D. Zhang and M. G. Amin was supported in part by a subcontract with Dynetics, Inc. for research sponsored by the Air Force Research Laboratory (AFRL) under Contract FA8650-08-D-1303. Y. D. Zhang was also supported in part by the Air Force Office of Scientific Research (AFOSR) through the Air Force Summer Faculty Fellowship Program under contract number FA9550-09-C-0114.
[3]. For maneuvering targets, high-resolution time-frequency analyses have been shown effective in resolving the multi-component Doppler signatures, and thus revealing rich and important information about the relative target altitude [4]-[7]. Estimation of the actual instantaneous target altitude, however, has not been considered within the non-stationary signal analysis framework.

In this paper, we develop a new altitude tracking technique of maneuvering targets in an MIMO radar environment. Only the array apertures in the range direction, which allow for spatial processing in the elevation dimension, is considered for presentation convenience. The proposed technique is based on recent advances in non-stationary signal analyses for instantaneous multi-component Doppler signature estimations (see, e.g., [6]-[10]). While these techniques stem from the earlier work on parameter estimation of multicomponent polynomial phase signals $[11,12]$, they enable instantaneous frequency estimation for more complicated signals which, as a whole, are difficult to parameterize. To obtain the absolute target altitude as well as the elevation maneuvering direction (ascending or descending), the target positions are estimated using the extended Kalman filer (EKF) that exploits the multi-component Doppler signature estimates. Different hypotheses of the initial conditions, including the initial target altitude and the maneuvering direction, are used and the maximum a-posteriori (MAP) criterion is applied to estimate these initial conditions.

Notations. A lower (upper) case bold letter denotes a vector (matrix). $(\cdot)^{*},(\cdot)^{T}$ and $(\cdot)^{H}$ respectively denote complex conjugation, transpose, and conjugate transpose operations. $\dot{a}$ denotes the derivative of $a$ with respect to time. $\mathbf{I}_{N}$ expresses the $N \times N$ identity matrix. In addition, $\mathbb{C}^{N \times M}$ denotes the complete set of $N \times M$ complex entries.

\section{SIGNAL MODEL}

\subsection{OTHR Propagation Model}

Because our main interest lies in the target altitude estimation, for simplicity and without loss of generality, we only consider twodimensional (2-D) position and velocity (range direction $x$ and altitude direction $z$ ) in the Cartesian coordinates. The target states at slow-time index $t$ is described by the 2-D position and velocity as

$$
\tilde{\mathbf{x}}_{k}=\left[\begin{array}{llll}
x_{k} & z_{k} & \dot{x}_{k} & \dot{z}_{k}
\end{array}\right]^{T} .
$$

Correspondingly, only the array apertures that lie in the range direction is considered. The $N_{t}$-element transmit array and the $N_{r^{-}}$ element receive array, which are both linear with aperture extended in the $x$-axis, form a monostatic MIMO radar configuration Due to 
the presence of micro-multipath propagation, the combination of the direct path and the multipath in both forward and return links yields the following four combinations of the two-way propagation: path I $\left(l_{1}: l_{1}\right)$, path II $\left(l_{2}: l_{2}\right)$, path III $\left(l_{1}: l_{2}\right)$ and path $\operatorname{IV}\left(l_{2}: l_{1}\right)$.

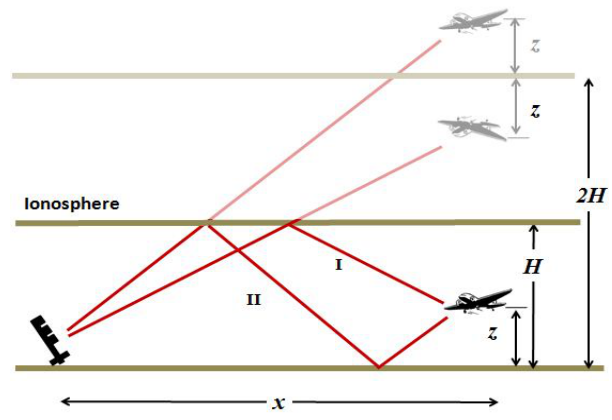

Fig. 1. Flat-earth model.

\subsection{MIMO Signal Model}

Denote $\mathbf{S} \in \mathbb{C}^{N_{t} \times T}$ as the narrowband waveform matrix which contains orthogonal waveforms to be transmitted from the $N_{t}$ antennas over a pulse-repetition period of $T$ fast-time samples. We assume that the waveform orthogonality is achieved in the fast-time domain, and $\mathbf{S S}^{H}=\mathbf{I}_{N_{t}}$ holds. For the $k$ th slow-time pulse, the signal received at the $m$ th receive array element is expressed as

$$
\mathbf{u}_{m, k}=\sum_{i=1}^{I} \sum_{n=1}^{N_{t}} \rho_{i, k} \mathbf{s}_{n} e^{j \phi_{i, m, k}+j \phi_{i, n, k}}+\mathbf{w}_{m, k},
$$

where $I=4$ is the number of multipath components, and $\rho_{i, k}$ is the complex response of the $i$ th multipath that accounts for target reflection and propagation delays. We assume that the clutter is sufficiently removed through, e.g., notch filtering of the low-frequency components. As such, elements of $\mathbf{w}_{m, k}$ in (2) can be considered as i.i.d. white complex Gaussian with $\mathcal{C N}\left(0, \sigma_{n}^{2}\right)$. The phase term $\phi_{i, n, k}$ is determined by the antenna position $d_{m}$ and is expressed as

$$
\phi_{i, m, k}=(2 \pi / \lambda) d_{m} \sin \left(\theta_{i, k}\right),
$$

where $\theta_{i, k}$ is the elevation angle of the $i$ th path. For paths I and II, the elevation angles of the target are shared by the transmit and receive arrays, and are respectively expressed as

$$
\theta_{1, k}=\tan ^{-1}\left(\frac{2 H-z_{k}}{x_{k}}\right), \quad \theta_{2, k}=\tan ^{-1}\left(\frac{2 H+z_{k}}{x_{k}}\right) .
$$

For path III, the direction of departure (DOA) is the same as $\theta_{1, k}$, and the direction of arrival (DOA) shares with $\theta_{2, k}$. For path IV, the DOD and DOA are respectively same as $\theta_{2, k}$ and $\theta_{1, k}$.

Multiplying $\mathbf{u}_{m, k}$ by $\mathbf{s}_{n}^{H}$ yields

$$
r_{m, n, k}=\sum_{i=1}^{I} \rho_{i, k} e^{j \phi_{i, m, k}+j \phi_{i, n, k}}+w_{m, n, k},
$$

where $w_{m, n, k}=\mathbf{w}_{m, k} \mathbf{s}_{n}^{H}$

Denote $\mathbf{a}_{i, k}=\left[e^{j\left(\phi_{i, 1, k}+\phi_{i, 1, k}\right)}, \ldots, e^{j\left(\phi_{i, N_{r}, k}+\phi_{i, N_{t}, k}\right)}\right]^{T} \in$ $\mathbb{C}^{N \times 1}$ as the steering vector of the virtual array corresponding to the $i$ th path, where $N=N_{t} N_{r}$, and let $\mathbf{A}_{k}=\left[\mathbf{a}_{1, k}, \ldots, \mathbf{a}_{I, k}\right]$. Further, denote $\boldsymbol{\rho}_{k}=\left[\rho_{1, k}, \ldots, \rho_{I, k}\right]^{T}$, and $\mathbf{w}_{k}=\left[w_{1,1, k}, \ldots, w_{N_{t}, N_{r}, k}\right]^{T}$. Then, the measurement data vector is expressed as

$$
\mathbf{r}_{k}=\left[r_{1,1, k}, \ldots, r_{N_{t}, N_{r}, k}\right]^{T}=\mathbf{A}_{k} \boldsymbol{\rho}_{k}+\mathbf{w}_{k} .
$$

\section{DOPPLER FREQUENCY MODEL AND ESTIMATION}

\subsection{Doppler Frequency Model}

To consider the Doppler frequency contained in $\rho_{i, k}$ in (2), we express $\rho_{i, k}$ as

$$
\rho_{i, k}=\sigma_{i, k} e^{-j 2 \pi \eta_{i, k} / \lambda},
$$

for $i=1, \ldots, I$, where $\lambda=c / f_{c}$ is the wavelength corresponding to carrier frequency $f_{c}$, and $\sigma_{i, k}$ represents the combined effect of transmit power, target reflection, which is a function of radar cross section (RCS), and the ionosphere as well as surface reflections of the $i$ th path at time index $k$. In addition $\eta_{i, k}$ is the two-way slant range, which is related to the multipath lengths as

$$
\eta_{i, k}=l_{u_{T, i}, k}+l_{u_{R, i}, k},
$$

where $l_{u_{T, i}, k}$ and $l_{u_{R, i}, k}$, respectively, denote the slant range of the forward and return links associated with path $i$. In particular,

$$
u_{T, i}=\{1,2,1,2\}, u_{R, i}=\{1,2,2,1\} \text {, for } i=\{1,2,3,4\} .
$$

The slant ranges $l_{1, k}$ and $l_{2, k}$ can be expressed in terms of the ground range $x_{k}$, the ionosphere altitude $H$, and the target altitude $z_{k}$, as

$$
l_{1, k}=\sqrt{x_{k}^{2}+\left(2 H-z_{k}\right)^{2}}, \quad l_{2, k}=\sqrt{x_{k}^{2}+\left(2 H+z_{k}\right)^{2}} .
$$

We use the following two variables as our observations:

$$
f_{\mathrm{ave}, k}=-\frac{1}{\lambda}\left(i_{1, k}+i_{2, k}\right), \quad f_{\mathrm{diff}, k}=\frac{1}{\lambda}\left|\dot{l}_{1, k}-\dot{l}_{2, k}\right| .
$$

Note in the above expression that the observation of Doppler frequency difference $f_{\text {diff }, k}$ is sign blind, implying ambiguity whether the target ascends or descends. $f_{\mathrm{diff}, k}$ can be expressed respectively for these two cases as

$$
f_{\text {diff }, k}= \begin{cases}-\frac{1}{\lambda}\left(i_{1, k}-i_{2, k}\right), & \text { target ascends } \\ -\frac{1}{\lambda}\left(i_{2, k}-i_{1, k}\right), & \text { target descends }\end{cases}
$$

Equations (10) and (11) are used for numerical evaluations of the slant ranges. Nevertheless, the following approximations, which hold true for $x_{k} \gg H \gg z_{k}$, reveal more insights of the relationship between the slant ranges and the other parameters:

$$
l_{1, k} \approx x_{k}+\frac{2 H^{2}-2 H z_{k}}{x_{k}}, \quad l_{2, k} \approx x_{k}+\frac{2 H^{2}+2 H z_{k}}{x_{k}} .
$$

In this case,

$$
f_{\mathrm{ave}, k} \approx-\frac{2}{\lambda}\left(1-\frac{2 H^{2}}{x_{k}^{2}}\right) \dot{x}_{k}, \quad f_{\mathrm{diff}, k} \approx \frac{4 H}{x_{k} \lambda}\left|\dot{z}_{k}\right|
$$

It becomes clear that the average Doppler component $f_{\text {ave }, k}$ is shared by all the four paths and reveals the target velocity in the range direction, whereas the small Doppler difference between the paths, $f_{\text {diff, },}$, is a function of $\dot{z}_{k}$. Therefore, for targets with vertical velocity, effective time-frequency analysis allows separation of the multicomponent Doppler signatures [6]. 


\subsection{Instantaneous Frequency Estimation and Signal Filtering}

As we described earlier, several techniques have been developed for the estimation of the instantaneous frequency of multi-component non-stationary signals with closely separated Doppler signatures. One of them is the signal stationarization technique, which is based on the local analysis of time, frequency, and phase coherence, and uses this information to merge local components in order to estimate the global time-frequency structures characterizing the signal $[5,6]$. We use this technique for IF estimation, and the details are omitted due to the space limitation.

Using the separated IF signatures, each multipath signal component can be separated as well. In particular, we are interested in signal components corresponding to paths I and II, which allow us to analyze the target maneuvering, in terms of the Doppler frequency and elevation angle, for each individual path. By multiplying the vector signal $\mathbf{r}_{k}$ by the conjugate of the phase estimate of the $i$ th path, $\exp \left(-j \psi_{i, k}\right)$ for $i=1$ and 2 , the signal associated with the $i$ th path is concentrated around the direct current (DC) component. We can design a narrowband filter to keep the $i$ th component and to filter out the other multipath components. Note that, unlike in the DOA estimation approaches [13] where only a single zero-frequency bin is used, we need to capture multiple frequency bins of the stationarized $i$ th component so as to keep the time-varying Doppler information, which is particularly important to determine the direction of the elevation velocity of the target. The captured signal vector is multiplied by $\exp \left(j \psi_{i, k}\right)$ to restore the Doppler information in each signal component., i.e.,

$$
\mathbf{r}_{k}^{[i]}=\exp \left(j \psi_{i, k}\right) \mathcal{P}\left[\mathbf{r}_{k} \exp \left(-j \psi_{i, k}\right)\right],
$$

where $\mathcal{P}$ denotes the filtering processing.

\section{TARGET ALTITUDE ESTIMATION}

The proposed technique take advantage of the resolved estimates of the Doppler signatures as well as the separated signal vectors individually corresponding to paths I and II. The incorporation of the instantaneous Doppler estimates generally provides good estimation of relative target altitude, but the instantaneous target altitude is still very sensitive to the initial target position vector $\tilde{\mathbf{x}}_{0}$, particularly the initial altitude $z_{0}$. In addition, as we discussed before, the Doppler difference has an ambiguity in the target direction of its elevation maneuvering.

To overcome these problems, we use multiple hypotheses of the initial target position and vertical orientation and find the best solution that maximizes the MAP criterion. Note that, due to the low SNR involved in this problem, the a-posteriori probability offered by the measured data at each time instant cannot reliably provide meaningful information. Rather, we use the a-posteriori probability of all the observed time instants so that a reliable MAP metric is achieved.

\subsection{Target State Model}

The target state vector, $\mathbf{x}_{k}$, consists of the target position vector, $\tilde{\mathbf{x}}_{k}$, and the complex response vector $\boldsymbol{\sigma}_{k}=\left[\sigma_{1, k}, \sigma_{2, k}, \sigma_{3, k}\right]^{T}$. Note that $\sigma_{4, k}$ is excluded from $\boldsymbol{\sigma}_{k}$ because it is considered identical to $\sigma_{3, k}$ due to propagation reciprocity. Therefore, we have

$$
\mathbf{x}_{k}=\left[\begin{array}{ll}
\tilde{\mathbf{x}}_{k}^{T} & \boldsymbol{\sigma}_{k}^{T}
\end{array}\right]^{T} .
$$

Then, the target state evolves according to the following linear stochastic model:

$$
\mathbf{x}_{k}=\mathbf{F} \mathbf{x}_{k-1}+\mathbf{v}_{k-1}
$$

where

$$
\mathbf{F}=\left[\begin{array}{ccc}
\mathbf{I}_{2} & \Delta \mathbf{I}_{2} & \mathbf{0} \\
\mathbf{0} & \mathbf{I}_{2} & \mathbf{0} \\
\mathbf{0} & \mathbf{0} & \mathbf{I}_{3}
\end{array}\right],
$$

and $\Delta$ is the pulse repetition interval (PRI). The process noise $\mathbf{v}_{k}$ is assumed to be zero-mean white Gaussian noise with covariance matrix $\mathbf{Q}$, which is assumed to be

$$
\mathbf{Q}=\left[\begin{array}{ccc}
\sigma_{p}^{2} \mathbf{I}_{2} & \mathbf{0} & \mathbf{0} \\
\mathbf{0} & \sigma_{v}^{2} \mathbf{I}_{2} & \mathbf{0} \\
\mathbf{0} & \mathbf{0} & \sigma_{g}^{2} \mathbf{I}_{3}
\end{array}\right]
$$

where $\sigma_{p}^{2}, \sigma_{v}^{2}$, and $\sigma_{g}^{2}$ are, respectively, the process noise variance for the position, velocity, and mode power level components of the state vector.

\subsection{Instantaneous Target Altitude Estimation}

In this step, the target altitude will be estimated with hypotheses of the initial target position, $\mathbf{x}_{0}$, and the direction of the target elevation velocity, i.e., ascending or descending. The ambiguities will be solved in the following sub-section.

In additional to the array data, the resolved Doppler estimates, which are related to the target states through (11), can be used as additional constraints. Because of the ambiguity of target vertical orientation as represented by the absolute value operation in (11), we divide the problem into two cases, i.e., when the target ascends or when it descends, and the Doppler difference is respectively expressed in (12). Such relationships can be treated as additional constraints or penalty functions to improve tracking performance [14, 15]. In this paper, however, we simply augment the Doppler estimates, $\mathbf{f}_{D, k}=\left[f_{\text {ave }, k}, f_{\text {diff }, k}\right]^{T}$, to be part of the observation vector of the maneuvering target at time instant $k$. As a result, the observation vector is defined as $\mathbf{z}_{k}=\left[\begin{array}{lll}\left(\mathbf{r}_{k}^{[1]}\right)^{T} & \left(\mathbf{r}_{k}^{[2]}\right)^{T} & \mathbf{f}_{D, k}^{T}\end{array}\right]^{T}$, which contains the array data individually separated for the two multipath modes and the Doppler signatures. The corresponding observation equation is expressed as

$$
\mathbf{z}_{k}=\mathbf{h}_{k}\left(\mathbf{x}_{k}\right)+\check{\mathbf{w}}_{k},
$$

where $\mathbf{h}_{k}\left(\mathbf{x}_{k}\right)$ collectively define the non-linear relationship between $\mathbf{z}_{k}$ and $\mathbf{x}_{k}$ as described in different parts of Section 3 , and $\breve{\mathbf{w}}_{k}$ represents the observation noise which is assumed to be zero mean and with variance matrix $\mathbf{R}_{n}$.

\subsection{MAP-based Initial State Estimation}

We attempt to solve the aforementioned two problems, i.e., the initial target altitude estimation and target elevation movement direction (ascending or descending), by making multiple hypotheses of the initial target position and vertical orientation and find the best solution that maximizes the MAP criterion. Define

$$
\mathbf{X}=\left[\mathbf{x}_{1}^{T}, \ldots, \mathbf{x}_{K}^{T}\right]^{T}, \quad \mathbf{Z}=\left[\mathbf{z}_{1}^{T}, \ldots, \mathbf{z}_{K}^{T}\right]^{T}
$$

to be the collection of the state and observation vectors, and

$$
\mathbf{X}^{\mathbf{x}_{0}, \nu}=\left[\left(\mathbf{x}_{1}^{\left[\mathbf{x}_{0}, \nu\right]}\right)^{T}, \ldots,\left(\mathbf{x}_{K}^{\left[\mathbf{x}_{0}, \nu\right]}\right)^{T}\right]^{T}
$$


to be the collection of the estimated state under the assumption of initial target position $\mathbf{x}_{0}$ and vertical orientation $\nu$, where $\nu \in[1,2]$ with 1 denoting target descending and 2 target ascending. In MAP estimation, the goal is to find $\hat{\mathbf{X}}$ that maximizes the posterior density, $f(\mathbf{X} \mid \mathbf{Z})$. Based on the Bayesian theorem, the $\hat{\mathbf{X}}$ that maximizes $f(\mathbf{X} \mid \mathbf{Z})$ also maximizes $\ln f(\mathbf{Z}, \mathbf{X})=\ln f(\mathbf{Z} \mid \mathbf{X})+\ln f(\mathbf{X})$. With the initial target altitude and moving direction in mind, we have

$$
f\left(\mathbf{X}^{\left[\mathbf{x}_{0}, \nu\right]}\right)=q_{0}\left(\mathbf{x}_{0}\right) \prod_{k=1}^{K} q\left(\mathbf{x}_{k}^{\left[\mathbf{x}_{0}, \nu\right]} \mid \mathbf{x}_{k-1}^{\left[\mathbf{x}_{0}, \nu\right]}\right),
$$

where $q_{0}\left(\mathbf{x}_{0}\right)$ is the pdf of the initial target altitude. On the other hand, $\ln f\left(\mathbf{Z} \mid \mathbf{X}^{\left[\mathbf{x}_{0}, \nu\right]}\right)$ is given by

$\ln f\left(\mathbf{Z} \mid \mathbf{X}^{\left[\mathbf{x}_{0}, \nu\right]}\right)=-\frac{1}{2} \sum_{k=1}^{K}\left(\mathbf{z}_{k}-\mathbf{h}_{k}\left(\mathbf{x}_{k}^{\left[\mathbf{x}_{0}, \nu\right]}\right)\right)^{H} \mathbf{R}_{n}^{-1}\left(\mathbf{z}_{k}-\mathbf{h}_{k}\left(\mathbf{x}_{k}^{\left[\mathbf{x}_{0}, \nu\right]}\right)\right)$

To find $\mathbf{x}_{0}$ and $\nu$ such that $\ln f\left(\mathbf{X}^{\left[\mathbf{x}_{0}, \nu\right]} \mid \mathbf{Z}\right)$ is maximized, the MAPbased initial state estimation can be expressed as

$$
\left[\hat{\mathbf{x}}_{0}, \hat{\nu}\right]=\arg \max _{\mathbf{x}_{0}, \nu}\left[\ln f\left(\mathbf{Z} \mid \mathbf{X}^{\left[\mathbf{x}_{0}, \nu\right]}\right)+\ln f\left(\mathbf{X}^{\left[\mathbf{x}_{0}, \nu\right]}\right)\right] .
$$

\section{SIMULATION RESULTS}

As an example, we consider a maneuvering target which makes a $360^{\circ}$ circular turn of radius $5 \mathrm{~km}$ in approximately $T=179.5 \mathrm{sec}-$ onds to descend by approximately 2,250 meters. The other key simulation parameters are listed in Table 1 . The target maintains a horizontal velocity of $175 \mathrm{~m} / \mathrm{s}$, and its altitude varies over time as

$$
h(t)=h(0)-\frac{v_{c, \max } T_{0}}{\pi}\left[1-\cos \left(\frac{\pi t}{T_{0}}\right)\right] .
$$

The corresponding Doppler signatures are depicted in Fig. 2.

Table 1. Key Parameters

\begin{tabular}{lcr}
\hline Parameter & Notation & Value \\
\hline initial range & $R(0)$ & $1,500 \mathrm{~km}$ \\
ionosphere height & $H$ & $160 \mathrm{~km}$ \\
target initial height & $h(0)$ & $10,000 \mathrm{~m}$ \\
horizonal target velocity & $v_{R, \max }$ & $175 \mathrm{~m} / \mathrm{s}$ \\
maximum descending velocity & $v_{c, \max }$ & $19.68 \mathrm{~m} / \mathrm{s}$ \\
carrier frequency & $f_{c}$ & $16 \mathrm{MHz}$ \\
waveform repetition frequency & $f_{s}$ & $40 \mathrm{~Hz}$ \\
\hline
\end{tabular}

We consider a MIMO array that consists of 6 transmit antennas and 10 receive antennas. Both arrays are minimum redundancy linear arrays extended in the range direction $[16,17]$. The unit interelement spacing is one wavelength. The transmit and receive arrays are separated by a $100 \mathrm{~km}$ cross-range distance. The input SNR at each antenna is $-10 \mathrm{~dB}$. For clutter removal, Signals falling in between $-1 \mathrm{~Hz}$ and $1 \mathrm{~Hz}$ are filtered out prior to the target tracking.

The estimated target positions are depicted in Fig. 3. In determining the target initial states, we assume that the initial target altitude is uniformly distributed between $5000 \mathrm{~m}$ and $15000 \mathrm{~m}$, and a step size of $50 \mathrm{~m}$ is used. Because the initial target altitude and the elevation motion direction are coupled, these two variables are jointly determined. As a result, the radar system can correctly identify that the target flies in a descending mode. In 100 Monte-Carlo trials with different noise realizations, the motion direction are all corrected identified. The average value of the estimated initial altitude is $9825 \mathrm{~m}$, and the standard deviation is $219 \mathrm{~m}$.

Fig. 3(a) shows the target altitude $\left(z_{k}\right)$, and the corresponding elevation velocity $\left(\dot{z}_{k}\right)$ is shown in Fig. 3(b). Overall, good instantaneous target altitude estimation is achieved. The initial target altitude estimate in this example is $9850 \mathrm{~m}$.

\section{CONCLUSION}

In this paper, we have developed a novel technique that provides a accurate estimation of the instantaneous altitude of a maneuvering target in an over-the-horizon radar system. By exploiting the recent non-stationary signal analysis techniques, the instantaneous frequency of the micro-multipath Doppler signatures can be resolved, and the received signal vector corresponding to each individual path can be obtained. These results allow significant performance improvement when tracking the target altitude through an extended Kalman filter. The proposed technique uses hypotheses of target elevation motion direction and initial target altitude, which are resolved using the maximum a-posteriori criterion. Simulation results verified the high-accuracy target position estimation results.

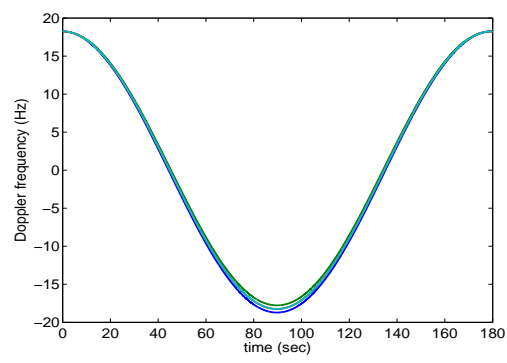

Fig. 2. Multipath Doppler signatures.

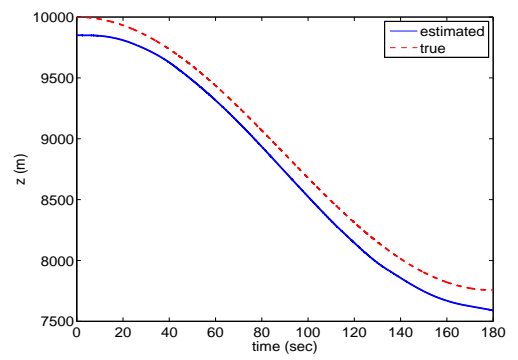

(a) Target altitude

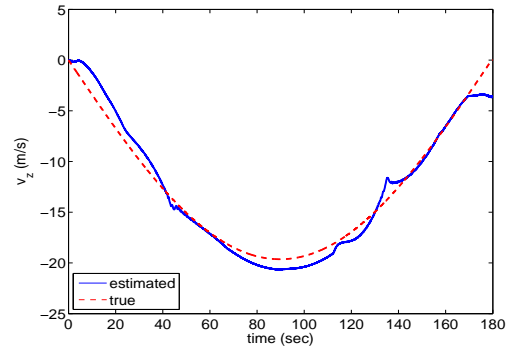

(b) Elevation velocity

Fig. 3. Tracking performance. 


\section{REFERENCES}

[1] J. M. Headrick and M. I. Skolnik, "Over-the-horizon radar in the HF band,” Proc. IEEE, vol. 62, pp. 664-?73, 1974.

[2] A. A. Kolosov, Over-the-Horizon Radar, Boston, MA: Artech House, 1987.

[3] R. H. Anderson, S. Kraut, and J. L. Krolik, "Robust altitude estimation for over-the-horizon radar using a state-space multipath fading model," IEEE Trans. Aerospace Electron. Syst., vol. 39, no. 1, pp. 192-201, Jan. 2003.

[4] Y. Zhang, M. G. Amin, and G. J. Frazer, "High-resolution time-frequency distributions for manoeuvring target detection in over-the-horizon radars," IEE Proc.-Radar Sonar Navig., vol. 150, no. 4, pp. 299-304, Aug. 2003.

[5] C. Ioana, M. G. Amin, Y. D. Zhang, and F. Ahmad, "Characterization of Doppler effects in the context of over-the-horizon radar," in Proc. IEEE Int. Radar Conf., Washington D.C., May 2010.

[6] C. Ioana, Y. D. Zhang, M. G. Amin, F. Ahmad, G. Frazer, and B. Himed, "Time-frequency characterization of micro-multipath signals in over-the-horizon radar," in Proc. IEEE Int. Radar Conf., Atlanta, GA, May 2012.

[7] C. Ioana, Y. D. Zhang, M. G. Amin, F. Ahmad, and B. Himed, "Time-frequency analysis of multipath Doppler signatures of maneuvering targets," in Proc. IEEE Int. Conf. Acoust., Speech, and Signal Proc. (ICASSP), Kyoto, Japan, March 2012.

[8] C. Ioana and A. Quinquis, "Time-frequency analysis using warped-based high-order phase modeling, EURASIP J. Applied Signal Proc., vol. 2005, no. 17, pp. 2856-2873, Sept. 2005.

[9] X. Li, G. Bi, S. Stankovic, and A. M. Zoubir, "Local polynomial Fourier transform: a review on recent developments and applications," Signal Proc., vol. 91, pp. 1370-1393, 2011.

[10] I. Djurovic, S. Djukanovic, M. G. Amin, Y. D. Zhang, and B. Himed, "High-resolution time-frequency representations based on the local polynomial Fourier transform for over-the-horizon radars," SPIE Radar Sensor Tech. Conf., Baltimore, MD, April 2012.

[11] S. Peleg and B. Porat, "Estimation and classification of polynomial-phase signals," IEEE Trans. Info. Theory, vol. 37. no. 2, pp. 422-430, March 1991.

[12] S. Barbarossa, A. Scaglione, and G. B. Giannakis, "Product high-order ambiguity function for multicomponent polynomialphase signal modeling," IEEE Trans. Sig. Proc., vol. 46, no. 3, pp. 691-708, March 1998.

[13] Y. D. Zhang, M. G. Amin, and B. Himed, "Direction-of-arrival estimation of nonstationary signals exploiting signal characteristics," in Proc. Int. Conf. Information Science, Signal Processing, and their Applications, Montreal, Canada, July 2012.

[14] D. Simon and T. L. Chia, "Kalman filtering with state equality constraints," IEEE Trans. Aerospace Electron. Syst., vol. 38, no. 1, pp. 128-136, Jan. 2002.

[15] K. Bell, "MAP-PF multi-mode tracking for over-the-horizon radar," in Proc. IEEE Radar Conf., Atlanta, GA, May 2012.

[16] J. A. Arsac, "Nouveau réseau pour l'observation radio astronomique de la brillance sur le soleil à $9359 \mathrm{MC}$," Compte Rend. Acad. Sci., vol. 240, pp. 942-945, Feb. 1955.
[17] A. T. Moffet, "Minimum-redundancy linear array," IEEE Trans. Antennas Propagat., vol. AP-16, pp. 172-175, March 1968. 